\title{
The Prohibitive Link between Position-based Routing and Planarity
}

\author{
David Cairns ${ }^{1}$, Marwan Fayed $^{1 *}$, Hussein T. Mouftah ${ }^{2}$ \\ ${ }^{1}$ Computing Science and Math, University of Stirling, Stirling, FK9 4LA, UK; ${ }^{2}$ SITE, University of Ottawa, Ottawa, \\ ON, K1N 6N5, Canada
}

\section{Abstract}

Position-based routing is touted as an ideal routing strategy for resource-constrained wireless networks. One persistent barrier to adoption is due to its recovery phase, where messages are forwarded according to leftor right-hand rule (LHR). This is often referred to as face-routing. In this paper we investigate the limits of LHR with respect to planarity. We show that the gap between non-planarity and successful delivery is a single link within a single configuration. Our work begins with an analysis to enumerate all node configurations that cause intersections in the unit-disc graph. We find that left-hand rule is able to recover from all but a single case, the 'umbrella' configuration so named for its appearance. We use this information to propose the Prohibitive Link Detection Protocol (PLDP) that can guarantee delivery over non-planar graphs using standard face-routing techniques. As the name implies, the protocol detects and circumvents the 'bad' links that hamper LHR. The goal of this work is to maintain routing guarantees while disturbing the network graph as little as possible. In doing so, a new starting point emerges from which to build rich distributed protocols in the spirit of CLDP and GDSTR.

Keywords: position-based routing, geographic routing, face routing, wireless routing

Received on 15 March 2013; accepted on 23 June 2013; published on 16 December 2013

Copyright (C) 2013 David Cairns et al., licensed to ICST. This is an open access article distributed under the terms of the Creative Commons Attribution license (http: / / creativecommons . org/licenses/by/3.0/), which permits unlimited use, distribution and reproduction in any medium so long as the original work is properly cited.

doi:10.4108/mca.1.3.e2

\section{Introduction}

The construction of network subgraphs appropriate for position-based (or geographic) routing protocols has, to date, remained a complex problem. These subgraphs are needed to recover from the local minima problem (see [3]) that prevents delivery and plagues positionbased protocols. Network subgraphs constructed for recovery using only 1 -hop information risk inaccuracies that cause routing failures $[15,24]$. One remedy is to allow nodes to cooperate. If permitted, cooperating nodes may construct a network subgraph that remedies any inaccuracies $[14,19,24,25]$. Yet the resources needed to power the many rounds of communication between nodes, risks being prohibitive in such a resource-constrained environment. The ideal wireless network subgraph would guarantee successful delivery while a) needing only 1-hop information and b) be able to acquire such information passively.

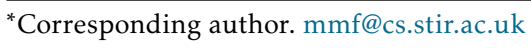

Traditionally, position-based routing protocols construct subgraphs (herein referred to as just 'graph') from available links in somewhat of a bottom-up fashion. Generally the idea is to extract a specific type of graph from the available nodes and links in the network. During the setup of such graphs each node evaluates available links to find those that preserve some global properties. Planar graphs [6] and k-spanners [23] are two such examples. The analogous question would be to ask, "what is the set of edges that must be preserved to guarantee a given feature in the graph?"

Our work is motivated by the opposite question, "What is the set of edges that must be deleted while still providing guarantees?" This work is a step in that direction. Without sacrificing the scalability and success of position-based routing, the goal of this work is to disturb the network as little as possible. To this end it is necessary to understand the causes for a positionbased routing protocol to fail to recover from local minima and deal with those causes directly. 
In this paper we investigate routing according to leftor right-hand rule (LHR). Using LHR, a node, upon receipt of a message, will forward to the neighbour that sits next in counter-clockwise order in the network graph. When used to recover from greedy routing failures, LHR guarantees success if implemented over planar graphs; for this reason it is often called 'facerouting'. We note, however, that if planarity is violated then LHR is only guaranteed to eventually return to the point of origin. Our work seeks to understand and correct the underlying causes of these failures.

We have chosen LHR for three reasons. First, it is most prevalent in position-based routing literature and hence well-studied. Second, it is a simple rule requiring little-to-no overhead. Finally, the ideal network graph remains elusive. To re-iterate, we envision the ideal graph as overcoming the inaccuracies that lead to routing failures; as one that results from knowledge of the 1-hop neighbourhood; and as one where each node transmits a constant number of messages.

We build on a provable enumeration of the possible types of intersections in a unit-disc graph (UDG), within which any two nodes are neighbours if separated by a maximum distance of one unit. Our analysis reveals that only three types of intersections are possible. A trace of face-routing over each intersecting neighbourhood further reveals that in only one of these configurations does LHR fail to recover: We call this the 'umbrella' configuration, so named for its appearance. The umbrella configuration naturally hides links and nodes from a face-routing traversal, partitioning the graph with respect to the traversal. Unless there appears some other non-local route to join these partitions, potential routes will be unavailable to any face-routing technique.

We use this information to propose the Prohibitive Link Detection Protocol (PLDP). PLDP identifies the umbrella configuration and removes from it a single link. In doing so, PLDP provides a graph over which any face-based method may provably guarantee delivery using standard geographic and face-routing rules.

In our evaluation we compare the setup and quality of PLDP graphs with CLDP and GDSTR using Netsim2 $[19,20]$. Our simulation results demonstrate that PLDP performance is similar to current facerouting schemes. Where PLDP separates itself is in the number of messages required to setup the network: Most nodes will need to generate no setup packets and from those that do, a very small number of packets is needed. Our evaluation will show that the small number of setup packets are indicative of the infrequency of the umbrella configuration, the consequence of which is that PLDP is able to preserve most of the links in the original network graph. In a manner of speaking our evaluations suggest that traditional face-routing schemes may be 'over-solving" the problem by planarizing networks.

On the surface this seems an unfair comparison since both GDSTR and CLDP operate without the unit disc assumption. We emphasize that our goal is to provide a better understanding of the underlying motivations for such distributed protocols and, in doing so, provide a new starting point for distributed protocols that may out-perform those of the current generation. PLDP may be unable to compete directly due to the unit disc assumption, but we will show that it provides a novel direction from which to build.

In summary, this paper seeks to provide a basis that is a lateral shift away from planarity so that better cooperative position-based protocols may be built. By investigating the underlying causes for failure, planarity is shown to be unnecessary in the majority of cases. We propose PLDP as a means to relax constraints on the network graph while preserving the promise of local face-routing techniques.

\section{Related Work}

Recovery algorithms in Euclidean position-based routing are equivalently known as face routing $[1,2]$ and perimeter routing [13]. Face routing was first proposed by Bose et al. in [1] with some theoretical bounds. Karp et al. independently proposed an identical mechanism in [13] but with work on a MAC-compatible implementation. Variants have since emerged addressing, for example, theoretical bounds in [16-18]. In [11], face-routing is augmented into a "select-and-protest" reactive protocol in order to reduce the information required to planarize the graph.

Wireless network graphs may consist of intersecting edges so it is necessary for planar subgraph methods to prune edges from the network graph so that it is planar while remaining connected. Gabriel Graphs (GG) and Relative Neighbourhood Graphs $(R N G)$ are planar graphs whose constructions are localised, a characteristic particularly suitable to sensor environments. Intersecting edges are eliminated by connecting pairs of nodes through witness nodes, if such a node exists in a common region. It has since been shown that 'Hello' messages may hinder network performance [10]. This is addressed in face-routing directly by [4] and more generally in [7, 9, 22]. Further work in [26] reduces the path length during the recovery phase.

These distributed constructions are unable to resolve links broken by obstacles or interference $[12,21]$. Recent breakthroughs have begun to surmount the impracticalities of face-routing while maintaining delivery guarantees $[14,19]$.

The Greedy Distributed Spanning Tree Protocol (GDSTR) algorithm in [19] builds on the fact that 
any message can be successfully delivered via depthfirst search if the network is connected via a spanning tree. This fact alone does not solve the problem: GDSTR provides optimizations to reduce the otherwise inefficient delivery requiring up to $2 n-3$ hops. The authors in [19] describe a new type of spanning tree, the hull tree, to route more efficiently. A hull tree is a spanning tree with one added piece of information: each node records the convex hull that contains all of its descendants in the tree. In GDSTR forwarding occurs greedily, as do most position-based protocols. If a message reaches a void, a recovery mode is initiated where convex hulls are used to determine the regions of the network that contain unreachable destinations. This information is used by GDSTR to route along the spanning tree to forward to the appropriate convex hull. If a node is found en route that is closer to the destination than the node where the message was stuck, then GDSTR returns to greedy forwarding. GDSTR is known to scale well as the neighbourhood size grows. Furthermore, the use of multiple hull trees adds faulttolerance to the network and if multiple trees are rooted at opposite ends of the network, routing efficiency improves.

The Cross-Link Detection Protocol (CLDP) proposed in [14], and later improved in [8], circumvents voids by face-routing. It uses left-hand rule over a planar subgraph of the network; its design however, is motivated by the observation that routing difficulties in planar subgraph methods arise, in part, due to the constructions themselves. (Recall from previous that successful local planar subgraph constructions rely on the unit disc graph.) For this reason, CLDP proposes an alternate construction of planar subgraphs that assumes only that links are bidirectional. CLDP operates in a distributed fashion, exchanging some localised operation for accurate information. The idea behind CLDP is that each node is able to probe the vicinity for intersecting links. A probe packet is initialised with the endpoints of the first link to be probed and forwarded according to left-hand rule. The probe eventually returns to its point of origin with a vector of the path taken. This information is shared with nearby nodes to prune links appropriately. To avoid the slow process of scheduling serial probes by neighbouring nodes, a system for concurrent probing is proposed. Concurrent probing is achieved by implementing a mechanism to 'lock' links so that no more than one link is removed at a time from any vicinity. CLDP is one of very few protocols to have been implemented on testbeds [14]. The associated communication complexities and storage costs revealed in this process (see $[15,19])$ are motivation to develop alternative approaches to guarantee delivery.

A more recent approach is to think about how the network might embed onto a different physical space.
One such work appears in the FaceTrace project [25] which imagines that nodes in the network sit on a highgenus topological surface, such as a torus. It is a novel technique that extracts onto these surfaces faces from the network itself, rather than faces associated with local minima in the network. In doing so, planarity emerges naturally. In simulation FaceTrace exhibits routing quality of a very high order but the setup cost is reported to be similar to those of GDSTR, numbering many orders of magnitude.

Protocols such as CLDP and GDSTR, in order to be feasible for physical networks, sacrifice efficiency for accuracy. CLDP requires high-complexity negotiations within each neighbourhood in order to prune appropriate links. GDSTR reduces the messaging complexity but must broadcast information to construct and maintain its hull trees. It remains an open question whether such trade-offs are a necessity. The work in [11] is a step in the right direction. Its recognition that there are available short-cuts when routing according to LHR is further evidence that the planarity assumption may be excessive.

By contrast, in this work we show that there exists a locally constructed, non-planar graph construction over which face-based protocols guarantee success.

\section{Links that Prohibit Routing Success}

In the previous section we noted that routing according to left-hand rule (LHR) alone, fails to provide a guarantee of success. Though this fact is well known, the reasons and circumstances under which delivery may fail are poorly understood. In this section we investigate the limits imposed by intersections on facebased recovery.

Our investigation begins with an enumeration of all of the types of intersections that may appear in the UDG. We focus this work on the unit disc graph (UDG), where all communication ranges are normalised. The UDG is appropriate since it limits potential routing options yet still poses a challenge to LHR routing.

\subsection{An Analysis of Intersection Types}

Consider any two intersecting edges. We provide the edges $a c$ and $b d$ in Figure 1 for reference. The nodes $a, b, c, d$ at the end points of these edges form a 4-gon (shown in Figure 1 using dashed lines). The question we ask is, which of the edges of the 4-gon may or may not be communicating links in the unit-disc graph? In order for at least one such edge to exist, we need to show that all four sides cannot be greater than both diagonals. Using cosine rule we know,

$$
(a c)^{2}=(a d)^{2}+(d c)^{2}-2(a d)(d c) \cos D .
$$

If $|a c|$ is less than or equal to 1 , then

$$
(a d)^{2}+(d c)^{2}-2(a d)(d c) \cos D \leq 1 .
$$




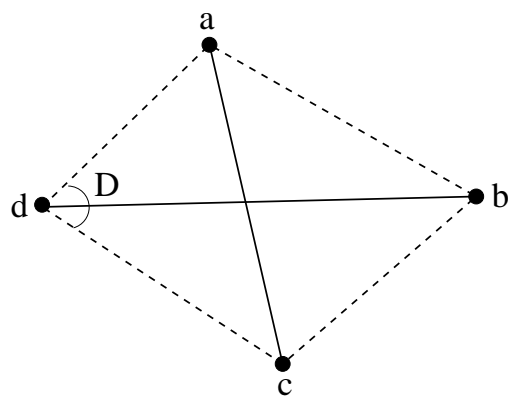

Figure 1. Intersecting links between two pairs of nodes may impose edges in a 4-gon.

When $D \geq \frac{\pi}{2}$, then $\cos D \leq 0$. In this case, $(a d)^{2}+$ $(d c)^{2} \leq 1$, which means $(a d) \leq 1$ and $(d c) \leq 1$. Thus, if an angle of the 4 gon is right or obtuse, then both incident edges must exist in the UDG. (By contrast, incident edges when $D<\frac{\pi}{2}$ may or may not exist.)

This implies and restricts the possible configurations that allow intersections to three in number, all shown in Figure 2. The two cases where the nodes of intersecting edges produce a 4-gon with two obtuse angles is shown in Figures $2 \mathrm{a}$ and $2 \mathrm{~b}$, while the 4-gon containing a single obtuse angle is shown in Figure 2c. (It is impossible for a 4-gon to be constructed with three obtuse edges; and that edges incident to an acute angle may or may not appear in the unit-disc graph.)

\subsection{The Prohibitive Link}

The finite and small number of possible intersections allows us to carefully examine the behaviour of a left-hand traversal over all possible cases. A left-hand traversal is deemed successful when it can identify a single unique face in an intersecting environment.

We show in Figure 3 the traces corresponding to the three intersections in Figure 2. Traversals are shown using a dotted line. In the first two cases an LHR traversal succeeds in identifying a single face irrespective of the point of entry into the intersecting environment. (We show via inductive proof in Section 4.2 that the same holds true when intersections are composed together.)

The 'bad' configuration occurs during a traversal of the intersection shown in Figure 3c. Here the different points of entry reveal that there are two faces with respect to LHR. This means there are two ways in which LHR may fail. The first is demonstrated by the dashed-dot-dash line originating at node $d$. (Entry at nodes $a$ and $b$ are analogous.) A traversal using leftor right-hand rule will never traverse edge ac while travelling through this intersection. Supposing $c$ must be traversed in order to reach the destination, LHR will fail. The second possible failure occurs when an LHR traversal encounters this intersection first via node $c$ in Figure $3 c$ using the dashed line. LHR traverses the inside of the triangle $\triangle a b d$ and exits without ever seeing edges that protrude from the outside of the triangle. As before, any such edges leading to the destination may be overlooked by an LHR traversal.

This represents the case where network node $a$ communicates with $b, c, d$, and $b$ with $d$; node $c$ communicates only with $a$. We call this case the umbrella configuration for its appearance.

The cause of both failures lies in the relationship between $\triangle a b d$ and $a c$ in Figure $3 c$ : There exists an edge from the triangle that is accessible only from inside the triangle. In other words, a traversal around the inside or the outside of the triangle fails to encounter all edges leading to the triangle. Both failures are solved by removing any of the edges that form the triangle. The easiest of these to identify and remove from the network graph is the edge of the triangle that forms the intersection in the umbrella configuration. In Figure $3 c$ this link is represented by $\overline{b d}$. We call this the prohibitive link.

The prohibitive link is most easily identified by the only node that is able to see all four nodes in the umbrella configuration. In Figure $3 c$ this responsibility falls on node $a$. It looks for intersections consisting of pairs links. The first link is formed by itself and an immediate neighour, with subsequent links formed between two immediate neighbours.

We revisit this subject and build a networking protocol in the next section. Before closing this section the outcome following a removal of the prohibitive link from the umbrella configuration is demonstrated in Figure 4. The intersection that was the umbrella configuration is reduced to a planar set of edges easily navigated by left- or right-hand rule.

\subsection{A Note on the Sufficiency of Prohibitive Links}

We note that it is sufficient to delete the set of prohibitive links while preserving delivery guarantees using face-routing schemes, yet it may be unnecessary to delete all of the links in the set. The minimal set of deletions remains an open problem.

An example of where it is sufficient but unnecessary to delete a link is shown in Figure 5. Node $a$ recognises prohibitive link $\overline{b d}$ in its neighbourhood. Removal of $\overline{b d}$ guarantees that all links in and out of the intersecting nodes will eventually be encountered. With respect to the local neighbourhood it is necessary to remove the prohibitive link. However the exitence of alternate paths outside of a neighbourhood, as shown in Figure 5 through nodes $p$ and $q$, suggest that it may be unnecessary to remove the prohibitive link.

We will see in our evaluations in Section 5 that the occurrence of umbrella configurations is so infrequent that this trade-off between global knowledge and local 


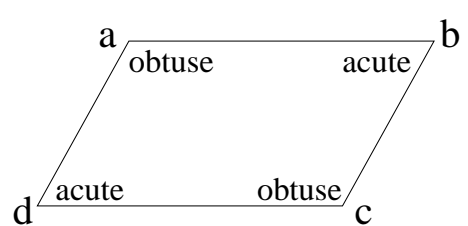

(a) All edges of the 4-gon must exist.

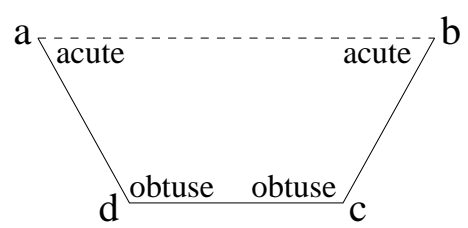

(b) Edges $b c, c d, d a$ must exist.

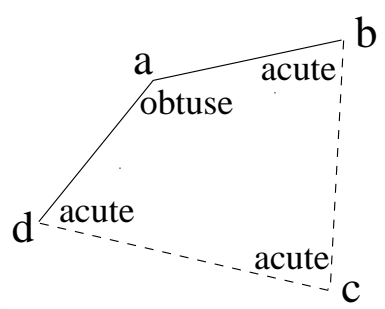

(c) Edges $a b$, ad must exist.

Figure 2. Possible 4-gons when edges intersect in the UDG; dashed lines indicating edges that may or may not appear.

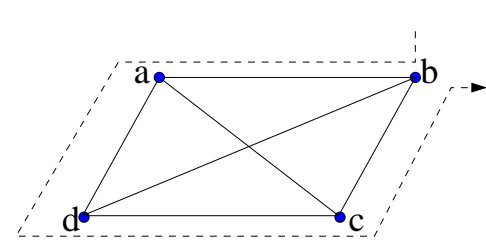

(a)

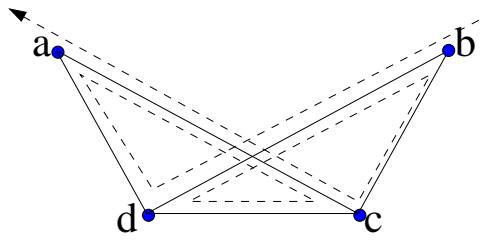

(b)

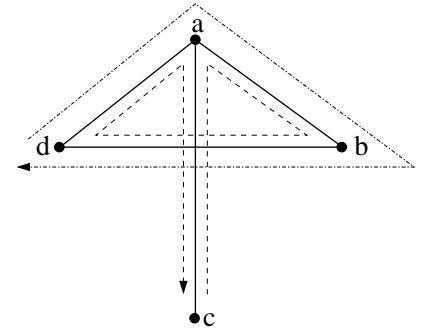

(c)

Figure 3. A unique face emerges from all but the 'umbrella' shape, shown in (c).

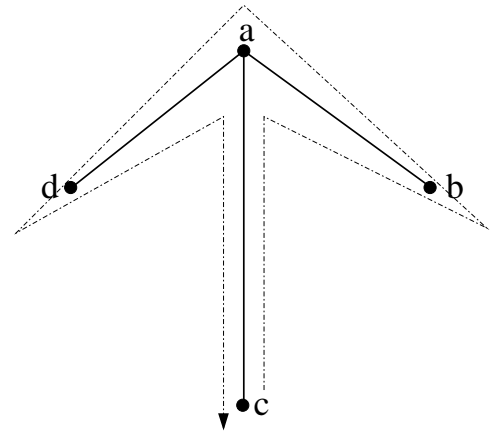

Figure 4. Removing prohibitive link $b d$ allows LHR to traverse all edges.

decisions may not even be worth considering. In the next section we use our knowledge of the prohibitive link to construct the prohibitive-link detection and routing protocol.

\section{Prohibitive Link Detection Protocol}

We have enumerated all possible intersections in the unit-disc graph and identified the type of intersection with the link that prohibits successful delivery when routing according to right- or left-hand rule. In this section we present a Prohibitive Link Detection Protocol (PLDP). Proofs of correctness may be found in [5].

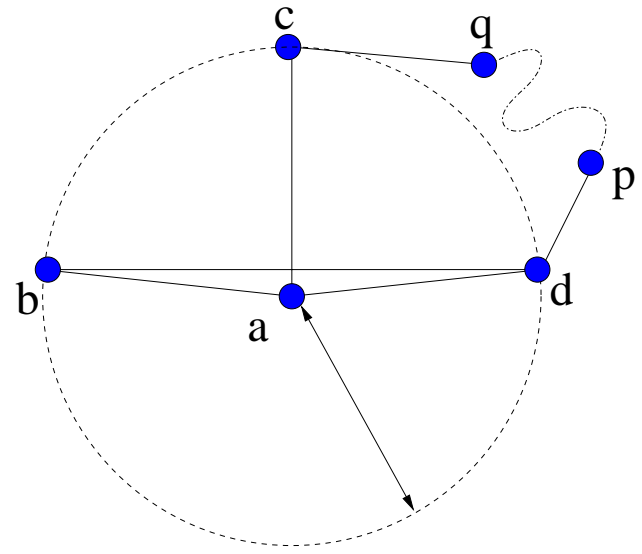

Figure 5. Removal of the prohibitive link is only sufficient.

\subsection{PLDP Overview}

We assume a static graph where each node is assigned a coordinate in a 2-dimensional Euclidean space. We assume that the graph is connected and that all links are bi-directional. PLDP functions adequately in a mobile space provided that changes in position occur over a greater time-frame than is required to re-evaluate local prohibitive links and transmit local updates. In this work communication range is fixed and uniform across all nodes.

The face-routing family of protocols preserve their delivery guarantees in PLDP graphs. During their normal operation nodes route in a greedy fashion 


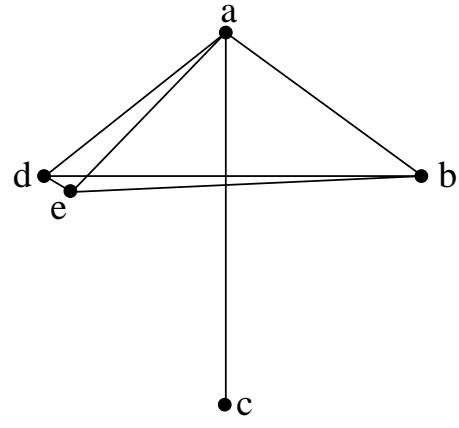

(a) Before detection phase.

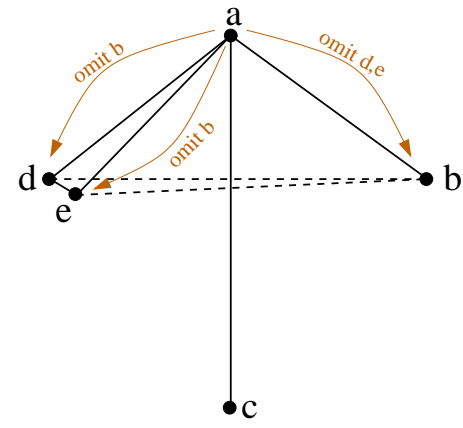

(b) Following detection phase links $d b$ and $e b$ omitted.

Figure 6. Local neighbourhood before and after the PLDP detection phase.

and forward messages to the neighbour that most reduces the distance to the destination. Where no such neighbour exists a message is deemed 'stuck' in a local minima and is forwarded according to left- (or right)hand rule. The node initially selected is the first to appear left (or right) of the line segment from the current location to the destination. The first node found that sits closer to the destination than the 'stuck' location returns to the greedy forwarding phase. Proofs of correctness and the ability to guarantee delivery to the destination appears in Section 4.2.

During the PLDP detection phase each node inspects its neighbourhood using neighbour positions reported in ordinary 'hello' packets. Each node evaluates intersections within range and flags any three neighbours that compose an umbrella configuration, as described in Section 3.2. Once sufficient information is compiled a node sends a notification packet to the neighbours that anchor prohibitive links.

Notification messages exchanged between nodes consist of either a delete or an insert instruction. As is suggested by its label, a delete deactivates a prohibitive link at the anchors of the link. Similarly an insert instruction reactivates a link previously deemed prohibitive. This allows for corrections as the network state changes.

We emphasize that PLDP takes a passive approach when looking for the recovery subgraph: In contrast to the 'active' approach taken by protocols such as CLDP and GDSTR, PLDP sends instruction messages only upon witnessing an umbrella configuration, and only to the neighbours that anchor the prohibitive link. The reduction in overall messaging is evaulated in Section 5.3.

The detection phase is demonstrated in Figure 6. In Figure $6 a$, node $a$ determines that two intersections in its vicinity contain prohibitive links, those links being $b d$ and $e b$. Nodes $b, d$, and $e$ have no knowledge of node $c$ 's existence. The responsibility falls on node $a$ to inform neighbours of their prohibitive links. Moving to Figure 6b, node $a$ instructs each of $d$ and $e$ to ignore their links to $b$ during recovery; similarly node $a$ instructs $b$ to omit links to $d$ and $e$.

Alternatively, notifications may be avoided entirely by producing and sending 'hello' notification packets that include neighbour information. Having been provided a 2-hop view of its neighbourhood a node can see all of the information it needs to identify prohibitive links (albeit at the cost of a larger 'hello' packet).

\subsection{Statement of Correctness}

Having identified and removed prohibitive links in umbrella configurations, we show in this section that PLDP will successfully route a message between two nodes if a path exists. We remind our reader that during the routing phase of PLDP, any standard position-based routing technique consisting of greedy + face-routing recovery may be implemented.

The following argument progresses first by defining the graph embedding so that we might state our claim. We first establish connectivity of the network embedding, and then show correctness by tracing a face-routing traversal within its intersections.

Definition 4.1. Let $G$ be an embedding of a graph, and $\operatorname{UDG}(G)$ be the unit-disc graph over $G$. We define $U m b(U D G(G))$ as the subgraph of $G$ where umbrella intersections are removed.

Proposition 4.2. If $U D G(G)$ is connected, then so too is $U m b(U D G(G))$.

Proof. We begin with an umbrella configuration in a unit disc graph. The prohibitive link is only removed when there is an alternate path remaining between all nodes in the neighbourhood. Thus, removal of the prohibitive link cannot disconnect the nodes in the umbrella configuration. Any connected component in $U D G(G)$ remains connected in $G^{\prime}$. 
Having shown connectivity we can now inspect traversals in the network embedding.

Proposition 4.3. We claim that in $G^{\prime}$, a traversal $T$, consisting of left-hand rule with memory, will find and traverse a unique face.

Proof. We prove by induction on the neighbourhoods witnessed by $T$. Consider the first neighbourhood, $k_{0}$, visible to starting node $v$. If no intersection is visible to $v$ then the next edge in $T$ is trivial. If, however, an intersection exists in $k_{0}$ then it must be in the form depicted in either of Figures 7a or 7b (see Section 3.1 for proof). We evaluate both cases below.

1. Consider the intersection in Figure 7a. For any $v \in\{m, n, o, p\}$ and destination $d$, if $\overline{v d}$ intersects with no local edges (ie. $\overline{v d}$ does not pass through quadrilateral (mnop)) then the next left edge - and thus first edge in the current face - is trivial. If, however, $\overline{v d}$ does pass through (mnop) as shown in Figure $7 \mathrm{a}$ then there are two cases:

$v=n$ The starting vertex is situated in the quadrilateral such that a single vertex sits left of $\overrightarrow{v d}$ and two vertices sit on the right. In Figure $7 \mathrm{a}$ this case is represented by $v=$ $n$. Node $n$ forwards to $m$. Both $m o$ and $m p$ intersect with $n d$, the line segment from the destination to the point where $T$ started, so $T$ will escape this neighbourhood when $m$ chooses the next CCW edge from $\overrightarrow{m p}$.

$v=o$ The starting vertex is situated in the quadrilateral such that a single vertex sits right of $\overrightarrow{v d}$ and two vertices sit on the left. In Figure 7a this case is represented by $v=o$. Here, too, $o$ forwards to $m$ and $m$ chooses the next CCW edge from $\overline{m p}$.

In either case, the face of interest begins at vertex $m$ where a cycle, if traversed, will be declared.

2. Consider the intersection in Figure $7 \mathrm{~b}$. Let starting node be $v \in q, r, s, t$ and destination $d$ sit such that $\overline{r d}$ intersects $\overline{q s}$ and $\overline{s d}$ intersects $\overline{r t}$. The trivial case is $v=q$. Three cases remain:

$v=r$ The starting vertex is situated in the quadrilateral such that a single vertex sits left of $\overrightarrow{v d}$ and two vertices sit on the right. In Figure $7 \mathrm{~b}$ this case is represented by $v=r . r$ forwards to $q$ where $T$ will escape the neighbourhood. (Recall that when $T$ intersects with $\overline{v d}, T$ switches faces.) In this case the cycle will be detected at $r$.

$v=s$ The starting vertex is situated in the quadrilateral such that a single vertex sits right of $\overrightarrow{v d}$ and two vertices sit on the left. In Figure $7 \mathrm{~b}$ this case is represented by $v=$ $s . s$ forwards along $\overline{s q}$ where $T$ escapes the neighbourhood. On its return, $T$ will detect a cycle at $s$ since node $t$ will avoid the edge $\overline{s q}$ since it was traversed previously.

$v=t$ The starting vertex is situated in the quadrilateral such that all three vertices sit left of $\overrightarrow{v d}$. In Figure $7 \mathrm{~b}$ this case is represented by $v=t$. Here, $T$ traverses $\overline{t r}, \overline{r q}$ before its escape from $q$. (Note that $\overline{q s}$ is not a valid edge since it intersects the edge previously traversed, $\overline{t r}$. $T$ will detect this cycle at node $t$.

Assume now that for any neighbourhood, $k_{i}$, traversal $T$ exits on the same face on which it enters. We show that for neighbourhood $k_{i+1}$ traversal $T$ exits on the same unique face on which it enters.

Referring once more to Figure 7, there are two types of neighbourhoods to consider. Those intersections whose endpoints join into a quadrilateral such as in Figure $7 \mathrm{a}$ require little consideration. For any entry point $m, n, o, p$ on the quadrilateral, $T$ will exit on the outside of this neighbourhood.

Similarly in Figure $7 b$, traversals entering on $\{q, r, s\}$ are trivial. We focus on traversals of $T$ that reach node $t$. From $t$ the next CCW edges in $T$ are $\{\overline{t r}, \overline{r q}\}$ since $\overline{q s}$ intersects $\overline{t r}$. From $q, T$ is forwarded along the next CCW edge.

Corollary 4.4. For any $G^{\prime}$, a traversal, $T$, consisting of lefthand rule with memory, guarantees a path will be found provided a path exists, or complete the face if no path exists.

Proof. We know that for a set of unique faces (ie. no intersections) in an embedding, that a left-hand traversal from source to destination is guaranteed to find a path provided one exists. Thus $T$, which finds sets of unique (non-intersecting) faces will find a path if it exists, or complete the face where no path exists.

Finally, we note that traversal $T$ requires no memory to succeed. A trace with no memory through all examples reveals that $T$ will escape from any intersecting neighbourhood via the same egress links as above. $T$ will achieve this by traversing the remaining links in the quadrilateral formed by the intersection.

\section{Simulation Results}

The previous section describes the PLDP protocol. We demonstrate the practical performance of PLDP via simulation in the sections that follow. 


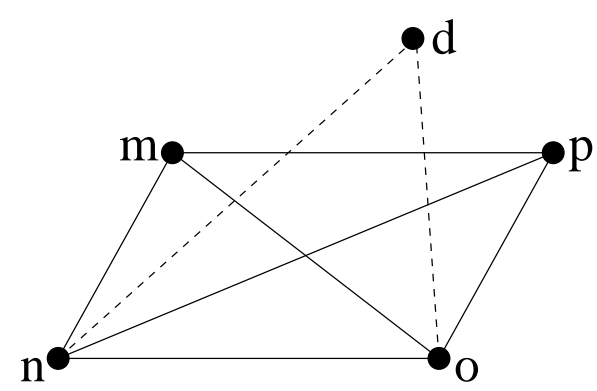

(a)

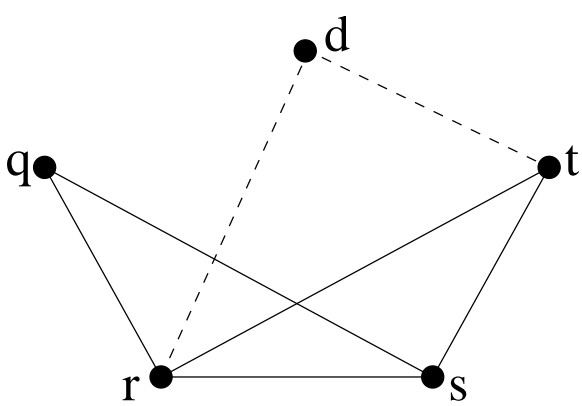

(b)

Figure 7. Once prohibitive links are omitted, two possible contentious configurations remain.

\subsection{Experimental Design}

So that we might better evaluate the performance of PLDP we have implemented PLDP into the netsim platform for geographic routing simulations used to evaluate GDSTR in [19] and GSpring in [20]. We compare PLDP primarily against two protocols. The first is CLDP $[14,15]$, a novel distributed planarization protocol that corrects for real-world events that violate the unit disc assumption. The second is GDSTR [19], also a distributed algorithm that reduces the high communication cost of CLDP but forces the network to cooperate as a whole.

The comparison of PLDP against CLDP and GDSTR may seem somewhat unfair given that CLDP and GDSTR operate outside of the UDG model. Our intention, rather than to 'compete' directly is to question the need for the one assumption on which all face-based routing techniques are based, that planarity is required for correct operation.

For completeness we set our experiments against a backdrop that includes more conventional face-routing schemes. Evaluations of the PLDP and CLDP network graph are made using GPSR [13] and GOAFR [16, 18]. GPSR design and accomplishments have served as the foundation on which later efforts have been built; it has long been considered the baseline for benchmark performance, while GOAFR provides some optimal theoretical bounds. Finally, GDSTR is implemented with two trees.

Simulation networks are composed of nodes placed uniformly in a space that is 1000 units squared; each node having a communication range of 10 units. Node density varies by increasing network size; neighbourhoods ranged from 4 to 16 nodes, on average. Each set of tests consisted of 5 runs, using the same five networks drawn from non-overlapping streams in each set of tests. A sample network with 250 nodes and an average degree of $\sim 8$ appears in Figure 8.

We test the validity of the CLDP and PLDP subraphs by routing with GPSR and with GOAFR.

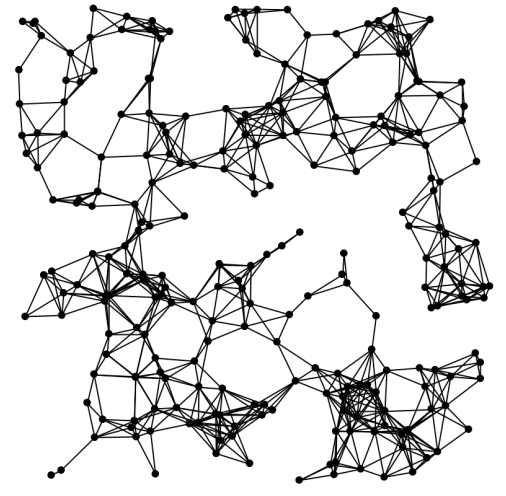

Figure 8. A sample network containing 250 nodes with an average degree of $\sim 8$.

Our primary performance metrics are hop stretch and message overhead. The latter takes our discussion into an investigation of the frequency of the umbrella configuration.

\subsection{Hop Stretch}

Hop stretch is defined as the ratio of hops taken vs hops of the minimum path. We consider only those paths over which packets were routed during facebased recovery; this is to avoid the distortion of results that would otherwise occur during the dominant greedy phase.

Observations for hop stretch are shown in Figure 9. In this figure we plot for PLDP with no changes to the routing protocols overtop, namely GOAFR and GPSR. The performance of both routing schemes is noticeably worse during the recovery phases over PLDP graphs than it is over planar graphs. In the best case scenario, routing over PLDP graphs during recovery takes $1 / 3$ rd greater number of hops than the next best scheme tested. Why is this, and is there anything that can be done?

To understand the cause we refer back to Figure $3 b$, in which we trace an LHR traversal. In this configuration 


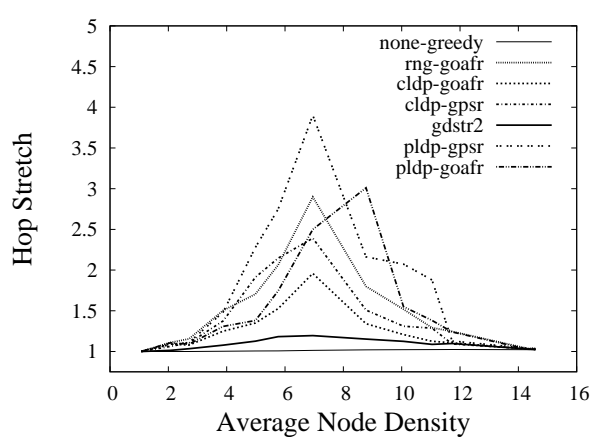

(a) Traditional routing schemes.

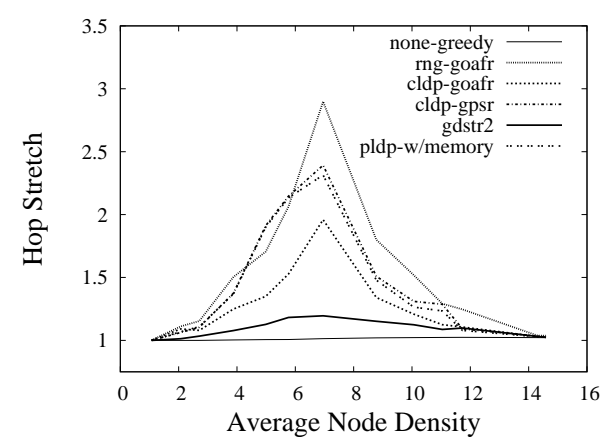

(b) After memory is added to GPSR packets.

Figure 9. Hop stretch of face-routing schemes on PLDP-induced graphs.

an LHR traversal that begins at node $b$ and exits at node $a$ requires 7 hops to escape. By contrast, were this region planarized then an LHR traversal requires 3 hops $^{1}$.

It is possible to resolve this issue by injecting memory into the routed packet. This memory consists of a record of the links traversed during face-routing so that LHR can avoid next hops that intersect with previous hops. In our evaluations up to this point we have tested unaltered routing protocols over the PLDP graph. Specifically this means that packets have no memory of where they have been. Thus many intersecting links will be traversed, only to return later along the same link in the opposite direction.

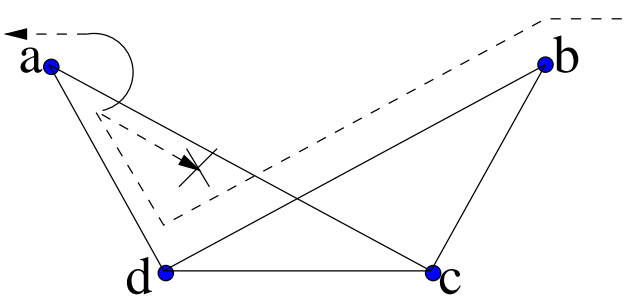

Figure 10. When packets record traversed links, nodes can substantially reduce hop counts.

So that our readers may clearly understand what is happening we point to Figure 10. A packet enters the intersecting region at node $b$ who, according to LHR forwards to node $d$, who then forwards to $a$. Node $a$ sees that the next link in counter-clockwise order $\overline{a c}$ intersects with link $\overline{b d}$, previously seen by this packet. In this example a packet escapes the intersecting region using 5 fewer hops than if the region had been planarized.

A noticeably different picture emerges if we record the recovery path and allow the routing protocol to

\footnotetext{
${ }^{1}$ The overall cost to a path stretch is much lower than would appear since the portion of time a packet sends in recovery is much lower than the portion of time that a packet sends in greedy mode.
}

skip past those links that would intersect previously traversed links. The effect of this "with memory" approach is demonstrated in Figure 9b, in which we plot GPSR with memory over a PLDP graph. Noting the change in range along the $y$-axis, we can see that the hop stretch along the PLDP graph has been diminished by roughly $1 / 2$.

In the next we evaluate the messaging cost associated with the setup and maintenance of the PLDP graph.

\subsection{Message Cost}

It is difficult, though necessary, to compare the setup of PLDP graphs with those setup by CLDP and GDSTR. The difficulty arises because of the difference in assumptions and goals: PLDP in its current form relies on the unit-disc assumption, whereas CLDP and GDSTR make none.

The comparison is necessary since, for all of their achievements, face-based protocols rely on the underlying assumption that some form of planarity is required for guaranteed delivery. PLDP graphs recognise that this assumption is stronger than necessary. Presumably, if we can reduce the set of undesired events then we can create more efficient realworld protocols.

The total number of messages sent in a network, on average, appears on log scale in Figure 11. The results obtained for CLDP and GDSTR are consistent with previous results: CLDP's relatively expensive messaging cost is reduced by an order of magnitude using the GDSTR approach. By contrast the number of PLDP packets produced is three orders of magnitude smaller than GDSTR. In our simulated networks PLDP produced close to zero packets until the average node density reached about 8 nodes. In the densest networks of approximately 16 nodes, 60 PLDP packets are sent.

In our trials these small numbers suggested that the number of prohibitive links is much smaller than we expected. In the next section we validate the small 


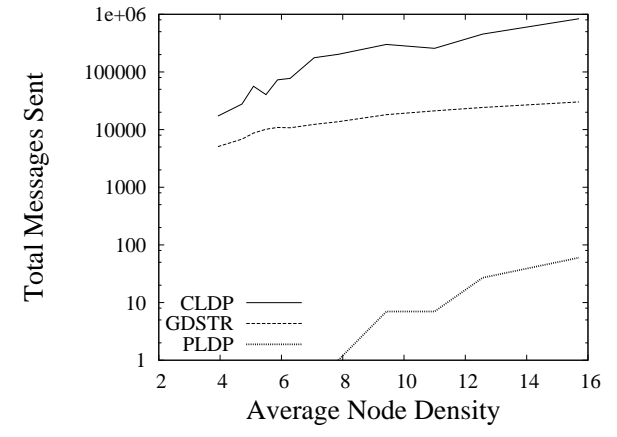

Figure 11. Packets sent or fowarded per node to achieve stability.

number of messages by investigating the frequency of prohibitive links.

\subsection{Umbrella Observations}

The very small number of PLDP packets produced implies that the number of prohibitive links is very small. To evaluate this hypothesis we generate large network graphs of varying density, distribution, and topology. Network nodes are distributed in a 200x200 unit space, each node with a fixed range of 8 units. We vary node density by changing the network size. Note that by changing size instead of communication range we can vary the density without affecting the maximum network diameter. Network sizes are 1500, 2500, and 3500 nodes. (In the uniform networks this results in average neighbourhood sizes of $\sim 7,12$, and 17 nodes.) To obtain results unbiased by isolated nodes we tabulate and experiment over the largest connected component of each network as described by Table 1 .

Table 1. Largest connected components in tested networks with $99 \%$ confidence intervals.

\begin{tabular}{||c|c|c|c||}
\hline Initial & \multicolumn{3}{|c||}{ Size of largest connected component } \\
\cline { 2 - 4 } Network Size & Uniform & Normal & Skewed \\
\hline 3500 & $3499.9 \pm 0.2$ & $3450.8 \pm 5.3$ & $3403.8 \pm 12.3$ \\
2500 & $2499.8 \pm 0.4$ & $2433.8 \pm 4.9$ & $2382.2 \pm 10.9$ \\
1500 & $1490.0 \pm 7.5$ & $1406.7 \pm 7.7$ & $1359.0 \pm 12.9$ \\
\hline
\end{tabular}

Nodes locations are chosen from a normal or skewed (Pareto) distribution in addition to the uniform distribution traditionally used to generate wireless network topologies. Uniformly distributed networks may be sufficient to provide insight yet are poor representations of many real deployments. Normal coordinates are generated with an average of 100 (the center) and a standard deviation of 40. Skewed coordinates are chosen from the Pareto distribution with scale parameter 1.0 and shape parameter 100.5. Example topologies appear in Figure 12.

In each network we count the number of intersecting links. From those we count the number of intersections that form the umbrella configuration. The results are tabulated and averaged in Table 2 with 99\% confidence intervals. The ratio of the two numbers appears in the last column, indicating that in all cases the proportion of intersections that form the umbrella configuration is slightly more than $1 \%$. This suggests that the frequency of configurations that might otherwise prevent successful delivery via LHR is quite small.

\section{Conclusions}

In this paper we have explored an new approach to graph construction for successful forwarding in position-based routing. It is instructive to compare this approach with previous work.

Traditionally, the success of face-routing schemes relies on the assumption that the underlying graph is planar. This is restrictive; local constructions of planar graphs risk inaccuracies, while co-operative (or global) constructions are resource intensive. In either case there has yet to appear an examination of the challenges that face left-hand rule in the presence of intersections.

By contrast, the approach taken in this work was to enumerate the configurations that form an intersection in the network graph. We then scrutinised each with a left-hand rule traversal so as to isolate the 'bad' configurations from which left-hand rule is unable to recover. In doing so we recognised the existence of a prohibitive link that has the potential to conceal other viable links from a left-hand rule traversal. We then presented PLDP, a protocol that detects and avoids the prohibitive link to successfully deliver packets. It operates locally and, unlike planarization methods, omits only essential links.

Our simulation results demonstrate that routing performance over PLDP graphs is similar to current face-routing schemes. Success rates over all graphs for all schemes is $100 \%$, while the path stretch in PLDP graphs is competitive with other methods. Where PLDP separates itself is in its messaging cost. Messages in PLDP are associated with the removal and maintenance of prohibitive links, which are shown to appear rarely. This suggests that traditional planar schemes may be 'over-solving' the problem.

We are working to release our source code as part of the Netsim 2 package. We are pleased to make it available upon request in the meantime. Currently we are working to remove the unit-disc assumption. Then, using the approach presented in this paper we expect to augment PLDP for general case networks where communication error and non-uniform range is commonplace. 


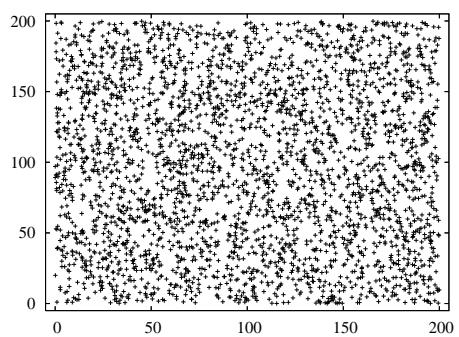

(a) A uniform network.

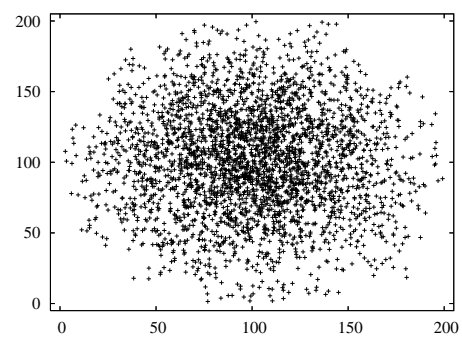

(b) A normal network.

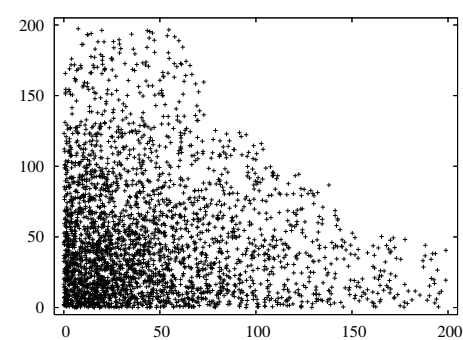

(c) A skewed (Pareto) network.

Figure 12. Example networks of 3000 (density $\simeq 7$ ) nodes with varying topologies.

Table 2. The number of umbrellas in tested networks with $99 \%$ confidence intervals.

\begin{tabular}{||c|c|c|c|c||}
\hline Size (Density) & Node Distribution & Intersections & Umbrellas & Ratio U/I \\
\hline & uniform & $119536.4 \pm 9563.5$ & $1586.4 \pm 96.1$ & 0.013 \\
\multirow{3}{*}{$1500(7.5)$} & norm & $2275283.8 \pm 226415.4$ & $30521.2 \pm 3360.0$ & 0.013 \\
& skew & $11577261.2 \pm 8833878.2$ & $130028.6 \pm 97577.0$ & 0.011 \\
\hline \multirow{2}{*}{$2500(12.5)$} & uniform & $939384.8 \pm 38816.3$ & $12454.6 \pm 802.6$ & 0.013 \\
& norm & $16631429.2 \pm 1775319.2$ & $225528.4 \pm 22547.8$ & 0.014 \\
& skew & $90216371.2 \pm 51567741.3$ & $1007242.4 \pm 546546.8$ & 0.011 \\
\hline \multirow{3}{*}{$3500(17.5)$} & uniform & $3692688.2 \pm 124431.4$ & $49094.8 \pm 1984.6$ & 0.013 \\
& norm & $67160718.8 \pm 4266681.4$ & $900722.2 \pm 58436.6$ & 0.014 \\
& skew & $280116248.2 \pm 97476260.3$ & $3199356.0 \pm 1024083.1$ & 0.012 \\
\hline
\end{tabular}

\section{References}

[1] Bose, P., Morin, P., Stojmenović, I. and Urrutia, J. (1999) Routing with guaranteed delivery in ad hoc wireless networks. In Workshop on Discrete Algorithms and Methods for Mobile Computing and Communications (DialM).

[2] Bose, P., Morin, P., Stojmenovic, I. and Urrutia, J. (2001) Routing with guaranteed delivery in ad hoc wireless networks. Wireless Networks 7(6): 609-616.

[3] Chen, D. and Varshney, P. (2007) A survey of void handling techniques for geographic routing in wireless networks. Communications Surveys \& Tutorials, IEEE 9(1): 50-67.

[4] Chen, D. and Varshney, P.K. (2007) On-demand geographic forwarding for data delivery in wireless sensor networks. Elsevier Computer Communications 30(14-15): 2954-2967.

[5] Fayed, M.M., Cairns, D.E. and Mouftah, H.T. (2010) An Analysis of Planarity in Face-Routing. Tech. Rep. TR-184, Computing Science \& Math, University of Stirling.

[6] Frey, H. and Stojmenovic, I. (2006) On delivery guarantees of face and combined greedy-face routing in ad hoc and sensor networks. In the 12th annual international conference on Mobile computing and networking (Mobicom): 390-401.

[7] Fubler, H., Widmer, J., Kasemann, M., Mauve, M. and Hartenstein, H. (2003) Contention-Based Forwarding for Mobile Ad-Hoc Networks. Elsevier's Ad Hoc Networks 1(4): 351-369.

[8] Govindan, Y.J.K.R., Karp, B. and Shenker, S. (2006) Lazy cross-link removal for geographic routing. In the 4th international conference on Embedded networked sensor systems (SenSys): 112-124.
[9] Heissenbãujtel, M., Braun, T., Bernoulli, T. and A, M.W. (2004) Blr: Beacon-less routing algorithm for mobile ad-hoc networks. ElsevierâĂ $\dot{Z}$ s Computer Communications Journal (Special Issue 27: 1076-1086.

[10] Heissenbüttel, M., Braun, T., Wälchli, M. and Bernouldi, T. (2007) Evaluating the limitations of and alternatives in beaconing. Elsevier Ad Hoc Networks 5(5): 558-578.

[11] Kalosha, H., Nayak, A., Ruhrup, S. and Stojmenovic, I. (2008) Select-and-protest-based beaconless georouting with guaranteed delivery in wireless sensor networks. In IEEE 27th Conference on Computer Communications (INFOCOM). (Pheonix, AZ, USA): 346-350.

[12] KARP, B. (2001) Challenges in geographic routing: Sparse networks, obstacles, and tra ffic provisioning, presented at DIMACS Workshop on Pervasive Networking.

[13] Karp, B. and Kung, H. (2000) Gpsr: Greedy perimeter stateless routing for wireless networks. In Proceedings of ACM MobiCom (Boston, MA).

[14] Kim, Y.J., Govindan, R., Karp, B. and Shenker, S. (2005) Geographic routing made practical. In Proceedings of the 2nd USENIX Symposium on Networked Systems Design and Implementation (NSDI) (Boston, MA, USA).

[15] Kim, Y.J., Govindan, R., KarP, B. and ShenKer, S. (2005) On the pitfalls of geographic face routing. In Proceedings of the 2005 joint workshop on Foundations of mobile computing (DIALM-POMC: 34-43.

[16] Kuhn, F., Wattenhofer, R., Zhang, Y. and Zollinger, A. (2003) Geometric Ad-Hoc Routing: Of Theory and Practice. In 22nd ACM Symposium on the Principles of Distributed Computing (PODC) (Boston, Massachusetts, USA).

[17] Kuhn, F., Wattenhofer, R. and Zollinger, A. (2002) Asymptotically Optimal Geometric Mobile Ad-Hoc Routing. In 6 th International Workshop on Discrete 
Algorithms and Methods for Mobile Computing and Communications (DIALM) (Atlanta, Georgia).

[18] Kunn, F., Wattenhofer, R. and Zollinger, A. (2003) Worst-Case Optimal and Average-Case Efficient Geometric Ad-Hoc Routing. In 4th ACM International Symposium on Mobile Ad Hoc Networking and Computing (MOBIHOC) (Annapolis, Maryland, USA).

[19] Leong, B., Liskov, B. and Morris, R. (2006) Geographic routing without planarization. In Proceedings of the $3 \mathrm{rd}$ USENIX Symposium on Networked Systems Design and Implementation (NSDI) (San Jose, CA, USA).

[20] Leong, B., Liskov, B. and Morris, R. (2007) Greedy virtual coordinates for geographic routing. In Proceedings of the IEEE International Conference on Network Protocols (ICNP).

[21] Lochert, C., Mauve, M., FÃIJÃßler, H. and rtenstein, H.H. (2005) Geographic Routing in City Scenarios. ACM SIGMOBILE Mobile Computing and Communications Review (MC2R) 9(1): 69-72.

[22] Sanchez, J., Marin-Perez, R. and Ruiz, P. (2007) Boss: Beacon-less on demand strategy for geographic routing in wireless sensor networks. In IEEE Internatonal Conference on Mobile Adhoc and Sensor Systems (MASS).: $1-10$.

[23] Schindelhauer, C., Volbert, K. and Ziegler, M. (2007) Geometric spanners with applications in wireless networks. Computational Geometry: Theory and Applications 36(3): 197-214.

[24] Seada, K., Helmy, A. and Govindan, R. (2004) On the effect of localization errors on geographic face routing in sensor networks. In Proceedings of the 3rd international symposium on Information processing in sensor networks (IPSN): 71-80.

[25] Zhang, F., Li, H., Jiang, A., Chen, J. and Luo, P. (2007) Face tracing based geographic routing in nonplanar wireless networks. In IEEE 26th Conference on Computer Communications (INFOCOM). (Anchorage, AK, USA).

[26] ZhaO, G., Liu, X., Sun, M.T. and MA, X. (2008) Energyefficient geographic routing with virtual anchors based on projection distance. Elsevier Computer Communications 31(10). 